\title{
The transition between the thick and thin Galactic disks
}

\author{
M. Haywood $\dagger$ \\ GEPI, Observatoire de Paris, CNRS, Université Paris Diderot; 92190 Meudon,France \\ email: Misha.Haywood@obspm.fr
}

\begin{abstract}
We study the transition between the thick and thick disks using solar neighbourhood data, focusing in particular on the status of local metal-poor thin disk stars $([\mathrm{Fe} / \mathrm{H}]<-0.3$ dex, $[\alpha / \mathrm{Fe}]<0.1 \mathrm{dex})$. The orbital properties of these stars, which are responsible for the hiatus in metallicity between the two disks, suggest that they most likely originate from the outer Galactic thin disk. It implies that the transition between the two stellar populations at a solar galactocentric distance must have occurred at a metallicity of about -0.3 dex. Transition stars at this metallicity are in fact present in local samples and fill the gap in $\alpha$-element between the thick and thin disks. These results imply that, at least from the local data, there is a clear evolutionary link between the thick and thin disks.
\end{abstract}

Keywords. Galaxy: abundances, Galaxy: disk, Galaxy: evolution, solar neighborhood

\section{Introduction}

The Galactic thick disk is, according to the local census, a population with characteristics neighbouring those of the thin disk : it is rotationally supported, with a somewhat higher internal kinematic dispersion, slightly metal-poor with an overlap in metallicity with this population. It is however possibly much older, and shows clear discontinuities with the thin disk, as visible in particular from metallicities. Several scenarios of the origin of this population have been put forward to explain these characteristics. The following three scenarious are often evoked: (1) the thick disk is an accreted population, (2) it is the first phase of the thin disk, heated up by an interaction episode with another galaxy (3) it could be the result of multiple, early mergers of gas rich subsystems from which the stars of thick disk would have formed. This last suggestion has been made by Brook et al. (2007) from numerical simulations where they apparently succeeded to produce a population with the main properties similar to those of the thick disk. In the first scenario, the properties of the thick disk are those of the accreted population. This is a difficulty, because while the range of satellite properties, encompassing the Milky Way is rather large, none of them approach those of the thick disk (in particular on the level of $\alpha$ elements). Furthermore, we have no direct clue to what these satellites may have been $10 \mathrm{Gyr}$ ago, and there is no evidence that a dwarf galaxy with properties similar to those of the thick disk may have formed at that epoch. In the second scenario, it is not entirely clear what exactly occurs in the transition phase (which may have lasted several Gyr) between the interaction with the satellite and the beginning of the thin disk phase, and, in particular, what discontinuities it may give rise to. However, while it is not clear what may differentiate the resulting thick disk scenario (2) and (3), a kind of parenthood is certainly expected with the thin disk, whereas no link is expected in scenario (1).

In the recent years, detailed spectroscopic data have demonstrated that the two disks

$\dagger$ Present address: GEPI, Observatoire de Paris-Meudon; 92190 Meudon,France 

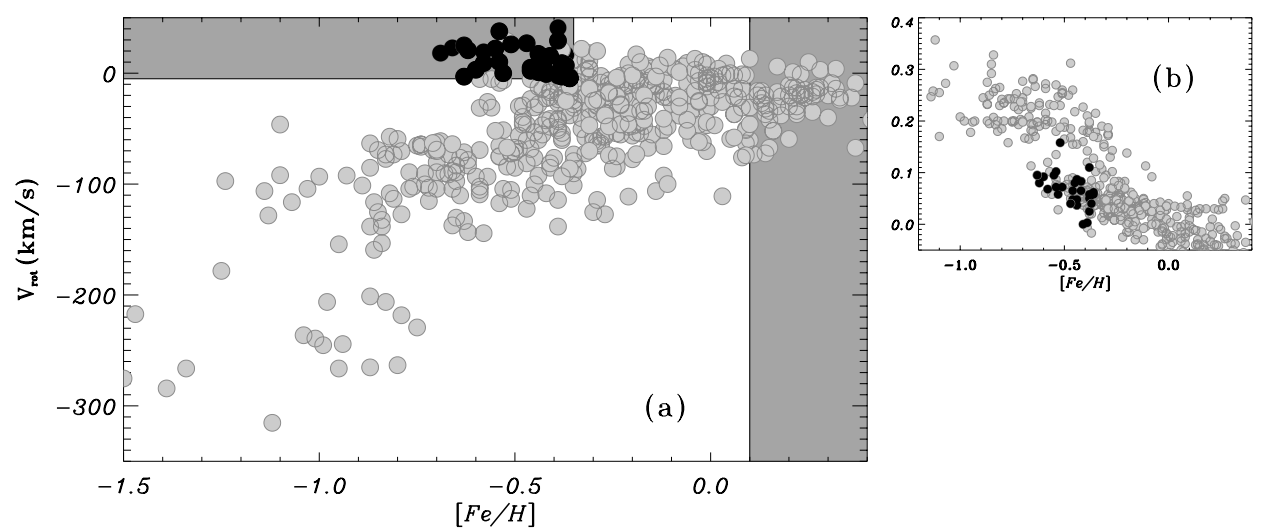

Figure 1. Galactic rotational velocity (relative to the Sun) as a function of metallicity and $[\alpha / \mathrm{Fe}]$ vs $[\mathrm{Fe} / \mathrm{H}]$ for the samples of Reddy et al. $(2003,2006)$ and Gilli et al. (2006). Black dots for stars with $[\mathrm{Fe} / \mathrm{H}]<-0.35$ dex and $\mathrm{V}_{\text {rot }}>-5 \mathrm{~km} . \mathrm{s}^{-1}$. Grey areas illustrate the two selections made on the catalogue of Nordström et al. (2004) and displayed in the next figure.

are apparently distinct in their chemical properties with two main differences : first, at a given metallicity, the $\alpha$-element content of the thick disk is offset by about +0.1 dex from the thin disk. Second, there is a hiatus of about 0.4 dex between the metal-rich stars of the thick disk (at $[\mathrm{Fe} / \mathrm{H}] \approx-0.2 \mathrm{dex}$ ) and metal-poor stars of the thin disk (at $[\mathrm{Fe} / \mathrm{H}] \approx-0.6 \mathrm{dex})$. The present study focuses on these two points, showing that these discontinuities are only apparent, and that a real continuity between the two disks does exist. The present results are obtained from the solar neighbourhood stars.

\section{Radial mixing in the Galactic thin disk}

Figure 1a shows the rotational velocity relative to the Sun of stars in the samples of Reddy et al. $(2003,2006)$ and Gilli et al. (2006) as a function of metallicity, while Fig. 1b shows $[\alpha / \mathrm{Fe}]$ vs $[\mathrm{Fe} / \mathrm{H}]$ for the same objects. On these plots, the thick disk stands out as a group enriched in $\alpha$ elements and lagging the LSR by about 50 to $150 \mathrm{~km} / \mathrm{s}$. In contrast, the metal-poor thin disk stars, (selected with $[\mathrm{Fe} / \mathrm{H}]<-0.35$ dex and $\mathrm{V}_{\text {rot }}>-$ $5 \mathrm{~km} . \mathrm{s}^{-1}$ ) rotate faster than the Sun. In order to understand the possible origin of this behaviour, we compare the orbital characteristics of the metal-poor and the metal-rich thin disk by selecting two subsamples from the catalogue of Nordström et al. (2004). The first includes stars with $[\mathrm{Fe} / \mathrm{H}]<-0.35$ dex and $\mathrm{V}_{\text {rot }}>-5 \mathrm{kms}^{-1}$, the other stars with $[\mathrm{Fe} / \mathrm{H}]>+0.1$ dex. Figure $2 \mathrm{ab}$ shows the distribution of apocentres $v s$ the pericentres for the two subsamples. Panel (a) shows that the metal-poor thin disk subsample is confined to the upper part of the plot, which is a consequence of the selection made on the rotational lag $\left(\mathrm{V}_{\text {rot }}>-5 \mathrm{kms}^{-1}\right)$. Panel (b) shows the metal-rich samples (selected only by metallicity), and clearly illustrates that this subsample populates mainly the inner orbits, or those orbits for which the mean orbital radius (defined as $\left(\mathrm{R}_{a}+\mathrm{R}_{p}\right) / 2$ ) is less than $8 \mathrm{kpc}$, in contrast to the metal-poor sample.

It has been suggested long ago that the stars are probably submitted to radial wandering caused by dynamical processes in the disk. A description of one such processes has been given by Sellwood \& Binney (2002), who found that the passage of spiral waves could result in a significant modification of the angular momentum of stars around corotation, with individual stars radially drifting by a few $\mathrm{kpc}$ in a few Gyr. Because this is a secular effect, we may expect to detect stars that are in the process of approaching 


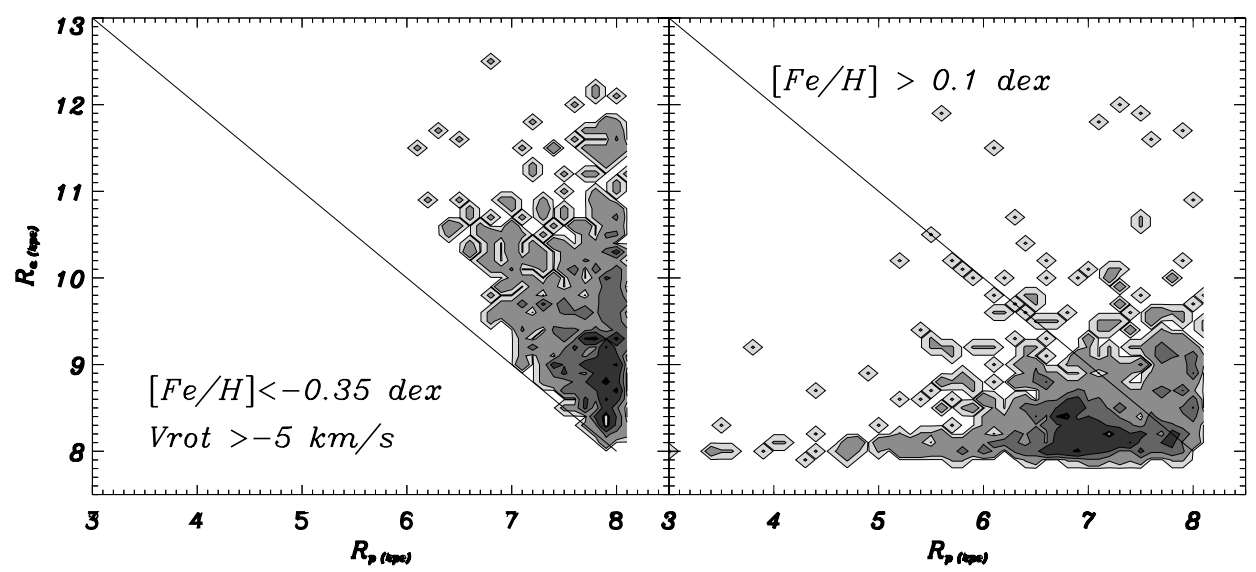

Figure 2. Distribution of apo- $v s$ pericentres for the two subsamples selected in the catalogue of Nordström et al. (2004). Left, metal-poor thin disk stars with $\mathrm{V}_{\text {rot }}>-5 \mathrm{~km} / \mathrm{s}$ and $[\mathrm{Fe} / \mathrm{H}]<-0.35$ dex. Right panel is for metal-rich stars with $[\mathrm{Fe} / \mathrm{H}]>+0.1$ dex.

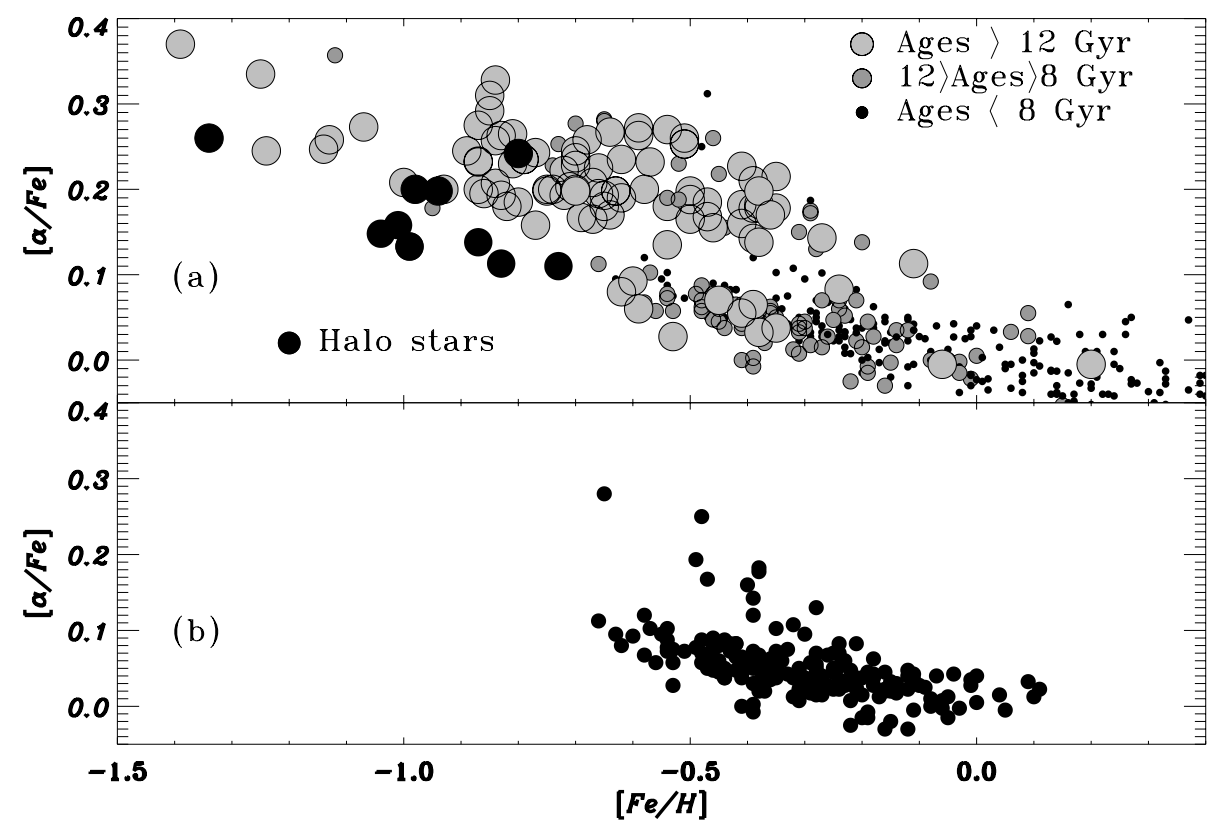

Figure 3. (a) Age distribution of stars in the $[\alpha / \mathrm{Fe}]$ vs $[\mathrm{Fe} / \mathrm{H}]$ diagram from Reddy et al. (2003, 2006) and Gilli et al. (2006). (b) Distribution of stars kinematically selected as thin disk stars in Reddy et al. (2006, their Fig. 12). Two branches are clearly visible, one made of metal-poor thin disk stars, the other of transition objects.

the solar orbit by moving outward or inward the Galactic disk, having orbital characteristics slightly off the main population. Combined with the fact that there exist a radial metallicity gradient of about $0.05-0.1 \mathrm{dex} / \mathrm{kpc}$ in the disk (the mean metallicity decreasing towards the outer disk), it is expected that metal-rich stars will be prevalent in the group of stars approaching the solar orbit from the inner disk, and metal-poor stars from the outer disk, as seen on Fig. 2. 


\section{The transition between the thick and thin disks}

If the above inferences are correct, then metal-rich stars (at $[\mathrm{Fe} / \mathrm{H}] \gtrsim 0.15 \mathrm{dex}$ ) and metal-poor stars of the thin disk $($ at $[\mathrm{Fe} / \mathrm{H}] \lesssim-0.3 \mathrm{dex})$ in the solar vicinity must be intruders from the inner and outer Galactic disks. It implies that the most metal-poor stars formed in the thin disk at solar galactocentric distance must have $[\mathrm{Fe} / \mathrm{H}] \approx-0.3$ dex. At what metallicity does the transition between the two disks occur? This is evidenced by the age-metallicity relation of solar neighbourhood stars (Haywood, 2006), which shows that the mean metallicity of the oldest thin disk stars is within $[-0.2,-0.3]$ dex - not -0.6 dex. Figure 3a shows an age-range of stars in the $[\alpha / \mathrm{Fe}]$ vs $[\mathrm{Fe} / \mathrm{H}]$ diagram. If we discard the metal-poor thin disk stars on the basis that they are not genuine solar galactocentric objects, there seems to be a clear age discontinuity between the two disks, with the thin disk being essentially younger than 8 Gyr and the thick disk older than 12 Gyr. In spite of the discontinuities visible in age and $[\alpha / \mathrm{Fe}]$, there is a real evolutionary link between the two populations. First, the thick disk terminates at the metallicity where the thin disk starts, at $[\mathrm{Fe} / \mathrm{H}] \approx-0.3$ dex. Most importantly, transition stars between the two disks do exist. In Fig. 12c of Reddy et al. (2006) (see Fig. 3b), kinematically selected stars of the thin disk in the $[\alpha / \mathrm{Fe}]$ vs $[\mathrm{Fe} / \mathrm{H}]$ diagram clearly show two branches, the first one made of metal-poor thin disk stars discussed above, the other towards the thick disk, starting at a metallicity of about -0.3 dex and with a higher level of $\alpha$-elements. In any case, statistics of solar neighbourhood stars show that transition objects are relatively rare, confirming that there is a gap in the star formation rate at that epoch in the Galaxy. A few of them are also found in Bensby et al. (2005) and Fuhrmann (2004), having [Fe/H] in the range $(-0.2,-0.4)$ dex.

\section{Microarsecond astrometry}

Virtually every aspect of the present study could be challenged by the expected returns of the space astrometric missions. Gaia in particular should provide samples of FGK stars to more than $1 \mathrm{kpc}$ from the Sun with astrometric accuracy sufficient for accurate age determination. The age-scale itself should also benefit from a drastic improvement from stellar physics. It is therefore expected that several million stars will be available for the kind of studies that are achievable today only with a handful of objects in the solar vicinity. It implies that, with the help of high resolution, high SN spectroscopic data, a detailed map of the interface between the thick and thin disk populations should be obtainable.

\section{References}

Bensby T., Feltzing S., Lundström I., \& Ilyin I., 2005, A\&A 433, 185

Brook C., Richard S., Kawata D., Martel H., \& Gibson B. K., 2007, ApJ 658, 60

Demarque P., Woo J.-H., Kim Y.-C., \& Yi S. K., 2004, ApJS 155, 667

Fuhrmann K., 2004, AN, 325, 3

Gilli G., Israelian G., Ecuvillon A., Santos N. C., \& Mayor M., 2006, A\&SA 449, 723

Haywood M., 2001, MNRAS 325, 1365

Haywood M., 2006, MNRAS 371, 1760

Nordström B., et al., 2004, A\&A 418, 989

Reddy, B. E., Tomkin, J., Lambert, D. L., \& Allende Prieto, C., 2003, MNRAS 340, 304

Reddy, B. E., Lambert, D. L., \& Allende Prieto, C., 2006, MNRAS 367, 1329

Sellwood, J. A., \& Binney, J. J., 2002, MNRAS 336, 785 